Research Article

\title{
Fasting Therapy Contributes to the Improvement of Endothelial Function and Decline in Vascular Injury-Related Markers in Overweight and Obese Individuals via Activating Autophagy of Endothelial Progenitor Cells
}

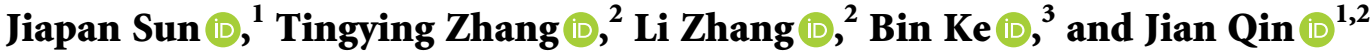 \\ ${ }^{1}$ Department of Traditional Chinese Medicine, The First Affiliated Hospital, Sun Yat-Sen University, Guangzhou, \\ Guangdong 510080, China \\ ${ }^{2}$ Department of Traditional Chinese Medicine, The Seventh Affiliated Hospital, Sun Yat-Sen University, Shenzhen, \\ Guangdong 518107, China \\ ${ }^{3}$ Department of VIP Ward, Sun Yat-sen University Cancer Center, Guangzhou, Guangdong 510060, China
}

Correspondence should be addressed to Jian Qin; himybox@yeah.net

Received 8 April 2020; Accepted 19 June 2020; Published 27 July 2020

Academic Editor: Raffaele Capasso

Copyright (c) 2020 Jiapan Sun et al. This is an open access article distributed under the Creative Commons Attribution License, which permits unrestricted use, distribution, and reproduction in any medium, provided the original work is properly cited.

\begin{abstract}
Background. High body mass index- (BMI-) related vascular injury contributes to the pathogenesis of the atherosclerotic cardiovascular disease (ASCVD). Rigorous calorie restriction is one of the major lifestyle interventions to reduce vascular risk in overweight or obese individuals. However, the effects of fasting therapy (FT) on vascular function and the mechanism are still unclear. This study was aimed to investigate the impacts of FT on endothelial function, arterial stiffness, and circulating arterial damage parameters in overweight and obese individuals and possible mechanism. Methods. Overweight and obese individuals participated in FT intervention (7-day very low calorie diet). Arterial function including brachial arterial flow-mediated dilation (FMD), brachial-ankle pulse wave velocity (baPWV), vascular injury-related markers including trimethylamine $\mathrm{N}$-oxide (TMAO), and leptin and endothelial microparticles (EMPs) were assessed. Endothelial progenitor cells (EPCs) of these participants were isolated and cultured to investigate EPCs function. mRFP-GFP-LC3 confocal microscopy scanning and western blot were carried out to determine autophagy. Results. After FT, body weight and BMI significantly decreased (81.76 \pm 12.04 vs. $77.51 \pm 12.06 \mathrm{~kg}, P<0.01 ; 29.93 \pm 2.82$ vs. $\left.28.47 \pm 2.83 \mathrm{~kg} / \mathrm{m}^{2}, P<0.01\right)$. FT remarkably improved FMD (5.26 \pm 1.34 vs. $6.25 \pm 1.60 \%, P=0.01)$ while baPWV kept unchanged. TMAO and leptin levels decreased $(3.96 \pm 1.85 \mathrm{vs.} 2.73 \pm 1.33 \mu \mathrm{mol} / \mathrm{L}$, $P=0.044 ; 6814 \pm 2639$ vs. $3563 \pm 2668 \mu \mathrm{mol} / \mathrm{L}, P<0.01)$. EMPs showed a decreased tendency. EPCs function was significantly improved, autophagy fluorescence intensity was enhanced, and the level of Beclin1, Atg5, LC3 II/I also increased after starvation in vitro, and the effects were blocked by autophagy inhibitor. Conclusions. Our present study demonstrated for the first time that FT markedly improves endothelial function and reduces the levels of arterial injury markers through improving EPCs function via activating autophagy. These findings provide a novel insight into FT as a lifestyle intervention strategy to promote the maintenance of vascular homeostasis in overweight or obese individuals. The trial was registered with ChiCTR1900024290.
\end{abstract}

\section{Introduction}

The prevalence of overweight and obesity is rising rapidly worldwide. High body mass index (BMI) is among the leading causes of elevated morbidity and mortality for atherosclerotic cardiovascular disease (ASCVD). Globally, it is estimated that 4 million deaths are caused by high BMI, more than two-thirds of which are related to ASCVD [1]. Impaired vascular function is the initial stage of ASCVD onset and predicts future adverse cardiovascular events [2]. Numerous reports support that overweight and obesity result in abnormal vascular function. Therefore, the 
maintenance of vascular function becomes an important target to reduce ASCVD in overweight and obese individuals [3-7].

Accumulating evidence indicates that the abnormal vascular function in overweight and obesity are mainly manifested as impaired endothelial function [4-6] and enhanced arterial stiffness [7] which can be evaluated by brachial arterial flow-mediated dilation (FMD) and brachialankle pulse wave velocity (baPWV). Moreover, circulating molecules such as trimethylamine $\mathrm{N}$-oxide (TMAO), endothelial microparticles (EMPs), and leptin, have been identified as biomarkers of vascular injury in obesity. TMAO is derived from gut microbiome metabolism of choline to trimethylamine, and EMPs are kind of vesicular structures shed from activated or apoptotic endothelial cells. Besides, leptin is a circulating hormone secreted by adipocytes that regulate food-intake and glycolipid metabolism. All these three acknowledged biomarkers are abnormally increased in obesity [8-15]. Hence, developing a therapeutic approach to synthetically improve endothelial function, arterial stiffness, and vascular injury-related markers might have important clinical implications in restoring vascular homeostasis of overweight and obese individuals.

Rigorous calorie restriction is an important lifestyle to reduce vascular risk and may help prevent the incidence of ASCVD in high BMI persons [16, 17]. Fasting treatment which can be carried out by drinking water only or having very low calorie diet has been proved to be an effective regimen to lose weight and restore metabolic disorders [18]. Our previous study demonstrated beneficial effects of fasting therapy (FT) on weight loss, insulin sensitivity, blood pressure, and lipid profiles in obese patients with metabolic disorders [19, 20]. However, to date, limited evidence is available to address the effect of FT on vascular function in overweight and obese individuals and possible mechanism.

Endothelial progenitor cells (EPCs), initially defined as circulating bone marrow-derived CD34-positive/KDR (kinase domain receptor)-positive cells [21], protect the physiological structure and function of the endothelium and maintain vascular homeostasis [22]. However, studies show that the depletion and dysfunction of the circulating EPCs are thought to be the foundation of vascular dysfunction in obesity [23-25]. Therefore, the upregulation of the functional potential of EPCs is a pivotal target to repair vascular function in patients with metabolic disorders.

Autophagy is a highly conserved physiological process controlling endothelial homeostasis in vascular beds [26]. Studies showed that autophagy defect results in endothelial dysfunction of patients with obesity and metabolic disorders, promoting the progression of ASCVD [26-28]. Fasting is a classical and acknowledged method to regulate autophagy [29-31], which may probably make contributions to EPCs function.

Based on the data mentioned above, we hypothesized that the FT would improve arterial function in overweight and obese individuals and reduce circulating vascular injuryrelated biomarkers via regulating autophagy to repair EPCs function. To address these assumptions, overweight and obese individuals who were enrolled in this pilot study received FT. FMD and baPWV as well as circulating arterial damage parameters such as TMAO, EMPs, and leptin, were evaluated. EPCs of these participants were isolated and cultured to investigate EPCs function and autophagy level after starvation. The present study may provide novel insight into the beneficial effects of FT as a lifestyle intervention strategy to maintain vascular homeostasis and retard the pathogenesis of high BMI-induced ASCVD.

\section{Subjects and Methods}

2.1. Subjects. Thirteen overweight or obese individuals $\left(\mathrm{BMI} \geq 25 \mathrm{~kg} / \mathrm{m}^{2}\right)$ aged between 25 and 65 were recruited to participate in this study from The First Affiliated Hospital, Sun Yat-Sen University. Exclusion criteria were persons with abnormal heart, liver, or kidney function, secondary hypertension, essential hypertension above the second grade, insulin use, severe gastric bleeding, cancers, blood diseases, active tuberculosis, and women during pregnancy, lactation or menstruation. All subjects were given written informed consent prior to study participation. Ethics approval was granted by the ethical committee of The First Affiliated Hospital, Sun Yat-Sen University in Guangzhou ([2019] 085). The trial was registered in Chinese Clinical Trial Registry of WHO (ChiCTR1900024290).

2.2. FT Intervention. The open-label, small sample size, selfcontrolled trial was conducted at The First Affiliated Hospital, Sun Yat-Sen University. Participants received the FT, blood samples were collected, and arterial function examination was taken at the first and the last day of FT (see Figure 1).

FT lasted for 7 days including 1 day with moderate calorie restriction (prefasting day, $<800 \mathrm{kcal} / \mathrm{d}$, fruits and vegetables), 5 days of intense calorie restriction (fasting period, $200 \mathrm{kcal} / \mathrm{d}$ ), and 1 day with the stepwise reintroduction of a normal diet (postfasting day, $800-1000 \mathrm{kcal} / \mathrm{d}$, semiliquid or semisolid food). Each subject was given 10-20 g of thenardite powder on the first day of the fasting period for bowel-cleansing. During the fasting period, patients were asked to drink at least $2 \mathrm{~L}$ of mineral water, have $200 \mathrm{kcal} / \mathrm{d}$ diet each day consisting of one serving of the liquid meal replacement and two cups of light vegetable soup, and receive L-carnitine (Lanling Pharmaceutical CO., LTD, Changzhou, China) 2 g, bid ( 9 a.m. and 4 p.m), i.v. infusion (dissolving $2 \mathrm{~g} \mathrm{~L}$-carnitine into $20 \mathrm{~mL}$ saline). Each serving of the liquid diet provided approximately $150 \mathrm{kcal}: 7.9 \mathrm{~g}$ protein, $2.8 \mathrm{~g}$ fat, and $22 \mathrm{~g}$ carbohydrate. The liquid diet was prepared by mixing the powdered product with $250 \mathrm{ml}$ water and was to be consumed at dinner. Two bowls of vegetable soup provided about $50 \mathrm{kcal}$ and were given at breakfast and lunch. All patients were required to engage in low-level physical activity consisting of $2 \mathrm{~h}$ of slow walking per day. Furthermore, participants were recommended to avoid alcohol, coffee, or tea during the study. All participants were compulsorily hospitalized during FT intervention $[19,20]$.

2.3. FMD Measurement. Each subject underwent vascular function examination by trained sonographers, performed between 8 and 11 a.m.; they were recommended to rest for 


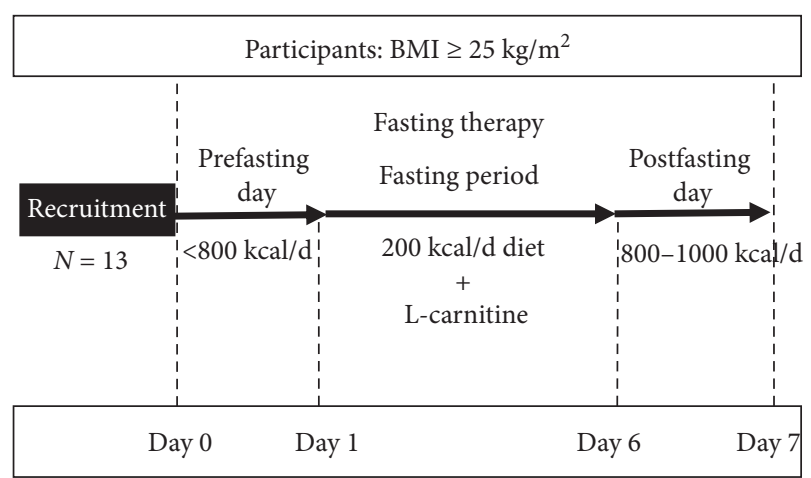

FIGURE 1: FT protocol. FT lasted for 7 days consisting of 3 phases (prefasting day, fasting period, and postfasting day). Measurements were taken at the time points of the $1^{\text {st }}$ and $7^{\text {th }}$ day of FT. FT: fasting therapy.

10 min or longer and were examined in a quiet, temperaturecontrolled room. FMD was examined through noninvasive ultrasound scan (UNEXEF38G, Sakae, Japan); it could dynamically record artery diameter, at rest (baseline) and reactive hyperemia period produced by using the cuff inflated to the greater of $50 \mathrm{mmHg}$ above systolic pressure or $200 \mathrm{mmHg}$ for $5 \mathrm{~min}$ as previously described [32]. FMD was calculated as follows:

$$
\operatorname{FMD}(\%)=\frac{\text { (maximum diameter }- \text { diameter at rest })}{(\text { diameter at rest })} * 100 \text {. }
$$

2.4. baPWV Measurement. Arterial stiffness was assessed with baPWV in supine individuals after rest. baPWV was measured by using an automatic device (VP-2000, Colin, Japan) as reported previously [33] and was expressed as centimeters per second $(\mathrm{cm} / \mathrm{sec})$. The measurement of baPWV is generally accepted as the simplest, robust, and reproducible method to represent arterial stiffness [34].

2.5. Biochemical Measures. Blood samples were collected between 08:00 and 10:00 in the morning after fasting overnight. TMAO was tested by using circulating plasma. Firstly, $10 \mu \mathrm{L}$ of internal standard solution (TMAO-d9, $5 \mu \mathrm{g}$ / $\mathrm{mL}$ ) was added to $100 \mathrm{ul}$ plasma; then $300 \mu \mathrm{l}$ of acetonitrile for protein precipitation was added, vortexed for $1 \mathrm{~min}$, centrifugated at $1000 \mathrm{rpm}$ for 5 minutes, $4^{\circ} \mathrm{C}$; at last, we took $200 \mu \mathrm{L}$ of the supernatant in the vial for HPLC-MS/MS analysis [35]. Leptin was tested by using circulating serum through the ELISA kit (RayBiotech, USA). Absorbance was measured at $450 \mathrm{~nm}$ using a microplate reader (Tecan, Crailsheim, Germany) and leptin concentrations were calculated according to the standard curve. EMPs were tested by using circulating using flow cytometry as previously described [36, 37]. To exclude the possibility of the unintended measurement of platelet microparticles, EMPs were defined as microparticles positively labeled by CD31 and negatively labeled by CD42.
2.6. EPCs Culture. EPCs were isolated and cultured as previously described [38]. After $4 \mathrm{~d}$ culture, nonadherent cells were removed and endothelial cell basal medium-2 (EBM-2) was changed (Lonza, Swiss). Later on, the medium was changed every 3 days. After 3 to 4 weeks' culture, late EPCs were examined by using flow cytometry analysis to identify endothelial markers including CD31, CD34, and CD309 (BD Pharmingen) as previously described [39] and then were incubated in starvation medium with or without 3-methyladenine (3-MA, $5 \mathrm{mM}, 2 \mathrm{~h}$ ).

2.7. EPCs Migration In Vitro. EPCs were marked with a “+” on the wall of the six-well plates when there were $80 \%-90 \%$ adherent cells and washed with the PBS to remove the nonadherent cells; images were photographed by a light microscope at that time and after $12 \mathrm{~h}$ in the same position for analyzing. Images were obtained using a light microscope at $100 \mathrm{x}$ magnification.

2.8. EPCs Adhesion In Vitro. EPCs $\left(3 \times 10^{4}\right.$ cells $\left./ 100 \mu \mathrm{L}\right)$ were resuspended at $1 \mathrm{~mL}$ with EBM- 2 serum-free medium and added to a human fibronectin (FN)-incubated six-well plates. The cells were cultured at $37^{\circ} \mathrm{C}$ for $5 \mathrm{~h}$, and the nonadherent cells were washed out with PBS. After fixing the cells with $4 \%$ paraformaldehyde for $15 \mathrm{~min}$ and $0.3 \%$ crystal violet for another $15 \mathrm{~min}$, images were obtained using a light microscope at $100 \mathrm{x}$ magnification.

2.9. EPCs Tube Formation In Vitro. A growth factor-reduced Matrigel (BD Biosciences, USA) was dissolved at $4^{\circ} \mathrm{C}$, added to 96-well plates at $80 \mu \mathrm{l} /$ well, and then incubated at $37^{\circ} \mathrm{C}, 5 \% \mathrm{CO}_{2}$ for $1 \mathrm{~h} .3 \times 10^{4}$ EPCs were resuspended in EBM- 2 serum-free medium at $100 \mu \mathrm{L}$ and loaded on the top of the Matrigel. After $5 \mathrm{~h}$ of incubation, EPC tube formation was assessed by light microscopy and photographed at 40x magnification.

2.10. EPCs Starvation in Vitro. For starvation, EPCs were incubated in starvation medium including $20 \mathrm{mM}$ HEPES [pH 7.4], $1 \% \mathrm{BSA}, 140 \mathrm{mM} \mathrm{NaCl}, 1 \mathrm{mM} \mathrm{CaCl} 2$ and $1 \mathrm{mM}$ $\mathrm{MgCl}_{2}$ for $1.5 \mathrm{~h} \mathrm{[31]}$.

2.11. Autophagy Process Detection. EPCs were transfected with mRFP-GFP-LC3 adenovirus obtained from Hanbio (Biotech, Shanghai, China). Confocal fluorescence microscopy (ZEISS, German) was used to evaluate autophagy flux by counting the cells with GFP-LC3 (green) puncta, RFPLC3 (red) puncta, and GFP+/mRFP+ -LC3 (yellow) puncta.

2.12. Western Blot Analysis. Total EPC protein was extracted and quantified by RIPA Lysis Buffer (Beyotime Biotechnology, China) containing protease inhibitors (Roche) and BCA assay kit (I Thermo, USA) separately. Protein extracts were separated by SDS-PAGE, transferred to PVDF membranes (Roche, Indianapolis, IN, USA). The following antibodies were used: anti-Beclin1 antibody (1:1000; ImmunoWay, USA), anti-Atg5 antibody (1:1000; 
ImmunoWay, USA), rabbit anti-LC3 antibody ( $1: 1000$; Cell Signaling Technology, USA), and anti-ACTB antibody (1: 1000; Cell Signaling Technology, USA). Proteins were visualized with HRP-conjugated anti-rabbit IgG or antimouse IgG (1:3000; Cell Signaling Technology, USA), followed by the use of the ECL chemiluminescence system (Thermo). And the level of protein was analyzed by using Image $\mathrm{J}$.

2.13. Statistical Analysis. All results were expressed as mean \pm standard deviation (SD). The normal distribution of continuous variables was determined via the Shapiro-Wilk test. Two groups of normal distribution data were performed using the Students $t$-test and Wilcoxon test for nonnormal distribution data. Statistical significance of multiple groups was assessed by one-way ANOVA for normal distribution data and followed by Bonferroni test. They were two-tailed. $P<0.05$ represented statistically significant. All statistical analyses were performed in Statistical Package for Social Sciences version 23.0 software (SPSS Inc., Chicago, IL, USA).

\section{Results}

3.1. Baseline Characteristics and Changes after FT. A total of 13 subjects received FT intervention in this pilot study (see Table 1). Results showed that there were significant reductions in body weight $(81.76 \pm 12.04$ vs. $77.51 \pm 12.06 \mathrm{~kg}$; $P<0.01)$, BMI $\left(29.93 \pm 2.82\right.$ vs. $\left.28.47 \pm 2.83 \mathrm{~kg} / \mathrm{m}^{2} ; P<0.01\right)$ after FT; and bodyweight decreased approximately $4.0 \mathrm{~kg}$ in average after 7 days, besides fasting plasma glucose (FPG, $5.25 \pm 2.24$ vs. $3.90 \pm 0.52 \mathrm{mmol} / \mathrm{L} ; P=0.04)$ and systolic blood pressure (SBP, $135.8 \pm 17.39$ vs. $125.3 \pm 15.01 \mathrm{mmHg}$; $P<0.01)$. As for lipid metabolism, triglycerides (TG, $P=0.03$ ) decreased, whereas total cholesterol (TC, $P=0.01$ ), low density lipoprotein cholesterol (LDL-C, $P=0.01$ ) increased, but TC and LDL-C would return to baseline or even lower after getting back to normal diets (unpublished data); it may be due to the steatosis and energy supply during fasting time. The exact mechanism deserves further investigation. All the participants went through FT without hypoglycemia or other severe adverse events. No one showed liver or renal impairment.

\subsection{Endothelial Function and Arterial Stiffness Response to FT.} FMD and baPWV are the most reproducible measures to indicate endothelial function and arterial stiffness which are widely used in clinical research. It showed FT ameliorated endothelial function after 7-day intervention. Overall, FMD significantly increased $(5.26 \pm 1.34$ vs. $6.25 \pm 1.60 \%$, $P=0.01$; see Figures $2(\mathrm{a})$ and $2(\mathrm{c}))$. baPWV had no statistically significant change $(1416 \pm 168.5$ vs. $1449 \pm$ $201.6 \mathrm{~cm} / \mathrm{s}, P=0.22$; see Figures $2(\mathrm{~b})$ and $2(\mathrm{~d})$ ).

3.3. Circulating TMAO, Leptin, and EMPs Response to FT. TMAO, leptin, and EMPs all varied among different subjects at baseline (minimum TMAO: $1.71 \mu \mathrm{mol} / \mathrm{L}$, maximum TMAO: $7.79 \mu \mathrm{mol} / \mathrm{L}$; minimum leptin: $2332.45 \mathrm{pg} / \mathrm{mL}$,
TABLE 1: Subject characteristics before and after FT interventions.

\begin{tabular}{lccc}
\hline Subject characteristics & Pre-FT & Post-FT & $P$ value \\
\hline Age, y & $43.54 \pm 10.60$ & - & - \\
Male, $n$ & 5 & - & - \\
Female, $n$ & 8 & - & - \\
Overweight, $n$ & 8 & - & - \\
Obesity, $n$ & 5 & - & - \\
Body weight, kg & $81.76 \pm 12.04$ & $77.51 \pm 12.06$ & $<0.01^{* *}$ \\
BMI, kg/m & $29.93 \pm 2.82$ & $28.47 \pm 2.83$ & $<0.01^{* *}$ \\
MWeight, kg & - & $4.25 \pm 1.01$ & - \\
Percentage of weight & & & \\
loss, \% & - & $5.30 \pm 1.40$ & - \\
& & & \\
Heart rate, per min & $68.76 \pm 9.68$ & $70.92 \pm 9.34$ & 0.40 \\
SBP, mmHg & $135.80 \pm 17.39$ & $125.30 \pm 15.01$ & $<0.01^{* *}$ \\
DBP, mmHg & $80.54 \pm 12.86$ & $80.77 \pm 8.97$ & 0.91 \\
FPG, mmol/L & $5.25 \pm 2.24$ & $3.90 \pm 0.52$ & $0.04^{*}$ \\
TC, mmol/L & $5.25 \pm 0.94$ & $5.70 \pm 1.25$ & $0.01^{* *}$ \\
LDL-C, mmol/L & $3.17 \pm 0.96$ & $3.77 \pm 0.91$ & $0.01^{* *}$ \\
HDL-C, mmol/L & $1.31 \pm 0.52$ & $1.11 \pm 0.24$ & 0.27 \\
TG, mmol/L & $1.71 \pm 0.53$ & $1.24 \pm 0.38$ & $0.03^{*}$ \\
\hline
\end{tabular}

BMI: body mass index; SBP: systolic blood pressure; DBP: diastolic blood pressure; FPG: fasting plasma glucose; TC: total cholesterol; LDL-C: lowdensity lipoprotein cholesterol; HDL-C: high-density lipoprotein cholesterol; TG: triglycerides. Data are shown as $\mathrm{n}$ or mean $\pm \mathrm{SD}$. ${ }^{*} P<0.05$, ${ }^{* *} P<0.01$.

maximum leptin: $11962.49 \mathrm{pg} / \mathrm{mL}$; minimum EMPs: 609.20/ $\mu \mathrm{L}$ plasma, maximum EMPs: $20415.90 / \mu \mathrm{L}$ plasma; see Figures 3(a)-3(c)). Mean level of TMAO was significantly lower after FT $(3.96 \pm 1.85$ vs. $2.73 \pm 1.33 \mu \mathrm{mol} / \mathrm{L}, P=0.04$; see Figure 3(d)), as well as leptin $(6814 \pm 2639$ vs. $3563 \pm 2668 \mathrm{pg} / \mathrm{mL}, P<0.01$; see Figure 3(e)). There was a tendency to the reduction of EMPs; no statistical significance was found $(1675(862.4,9824)$ vs. $1697(747.7,4035) / \mu \mathrm{L}$ plasma, $P=0.38$; see Figure $3(\mathrm{f}))$.

3.4. Culture and Identification of EPCs. To investigate the effects of starvation on EPCs function, we isolated and cultured late EPCs from participants' peripheral blood. After about 3 to 4 weeks of culture, the morphology of late EPCs closely approximate mature endothelial cells (see Figure 4(a)). The markers of late EPCs were detected by flow cytometry (see Figure 4(b)), in which CD34 (48.4 $\pm 5.6 \%$ ), CD31 (86.3 $\pm 6.3 \%)$, CD309 $(88.2 \pm 7.1 \%)$ increased, and monocyte marker CD14 were significantly reduced $(2.3 \pm 0.4 \%)$; it was similar with the previous study.

3.5. The Effects of Starvation on EPCs Function In Vitro. EPCs were incubated in starvation medium for $1.5 \mathrm{~h}$. The migration $(P<0.01)$, adhesion $(P<0.01)$, and tube formation $(P<0.01)$ of EPCs were all improved compared with the nonstarvation group (see Figure 5). These data showed starvation ameliorated EPCs function.

EPCs were transfected with mRFP-GFP-LC3 adenoviruses to detect the autophagy process (see Figure 6). The fluorescence intensity of LC3 was enhanced in EPCs with starvation compared with nonstarvation group $(P<0.05)$, 


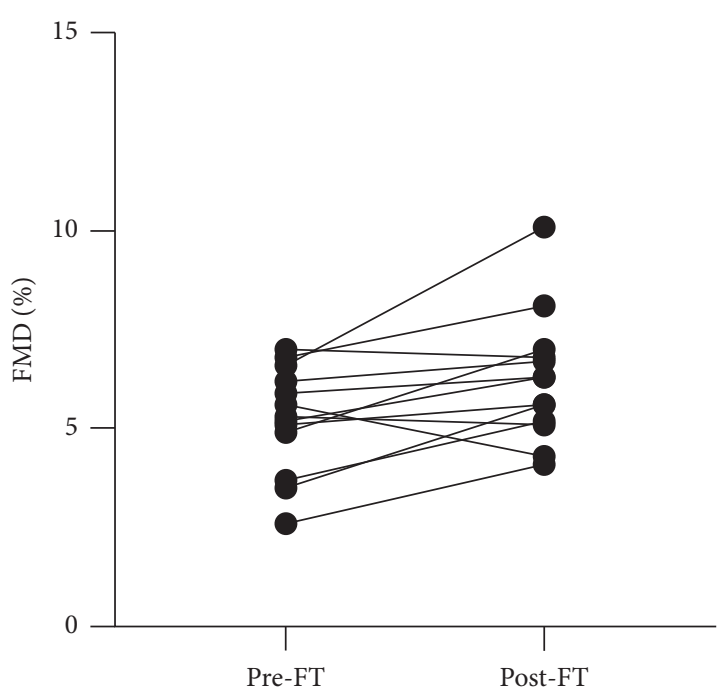

(a)

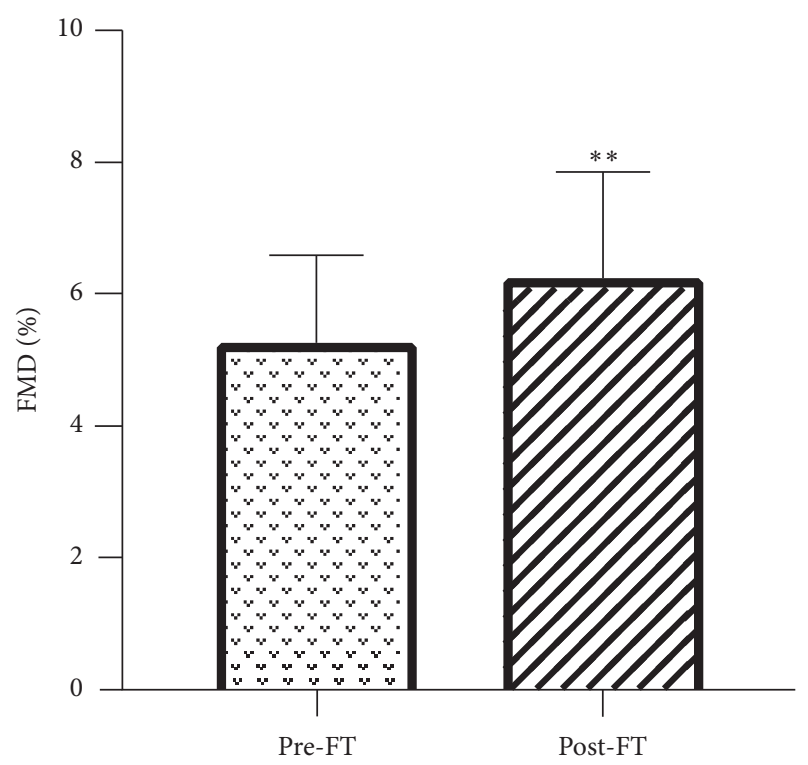

(c)

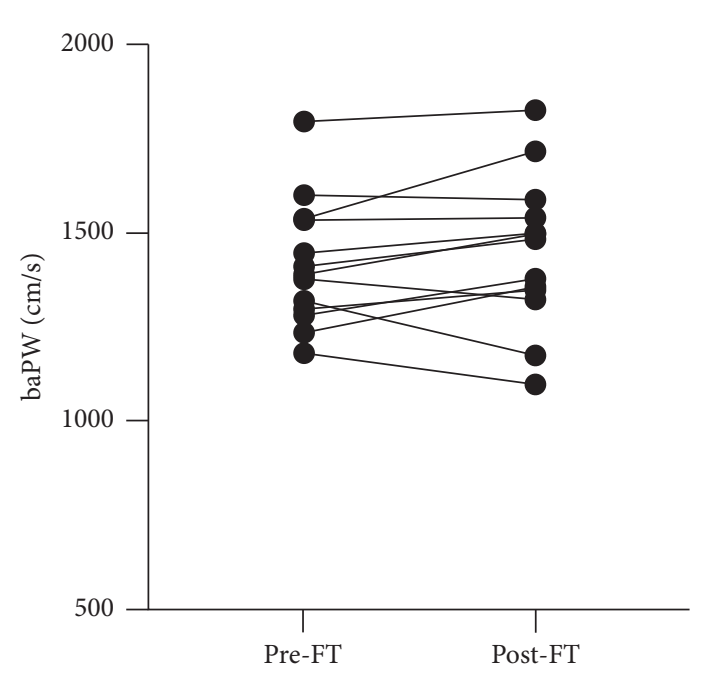

(b)

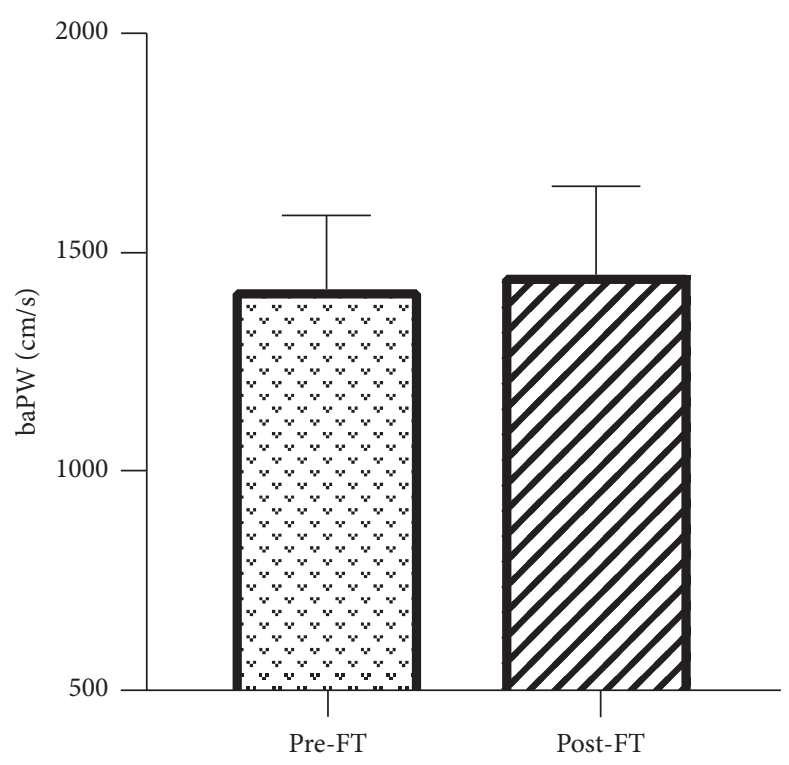

(d)

FIgURE 2: Effects of FT on FMD and baPWV. (a) Individual changes in FMD. (b) Individual changes in baPWV. (c) The mean value of FMD. (d) The mean value of baPWV. ${ }^{*} P<0.05,{ }^{* *} P<0.01$. FMD: flow-mediated dilation; baPWV: brachial-ankle pulse wave velocity.

paralleled with the increase of protein levels of Beclin1 $(P<0.01), \operatorname{Atg} 5(P<0.01)$, and LC3 $(P<0.01)$. In addition, 3 -MA, the autophagy inhibitor, blocks the effects of starvation in improving EPCs function (see Figure 5).

\section{Discussion}

This pilot study explored the effects of FT on arterial function, vascular injury-related biomarkers, and possible mechanisms in overweight and obese individuals. Apparently, FT safely and effectively improved FMD and reduced body weight among overweight and obese individuals, whereas baPWV showed no marked change. Meanwhile, FT reduced vascular injury-related circulating biomarkers such as TMAO and leptin, and EMPs exhibited a descending trend but with no statistical significance. In vitro, starvation improves EPCs ability of migration, adhesion, and angiogenesis via activating autophagy, and inhibition of autophagy eliminated the improvement of EPCs function. To the best of our knowledge, these data demonstrated for the first time that FT reduces vascular risk through improving endothelial function and reducing vascular injury-related biomarkers in overweight and obese individuals via upregulating EPCs function and autophagy level.

For overweight and obesity, calorie-restricted weight management is recommended as the cornerstone of staying healthy [40]. Fasting is a procedure that a person voluntarily goes without food for a certain length of time, living mainly on their fat reserves [18]. L-carnitine is a conditionally essential nutrient necessary for transporting long-chain fatty 


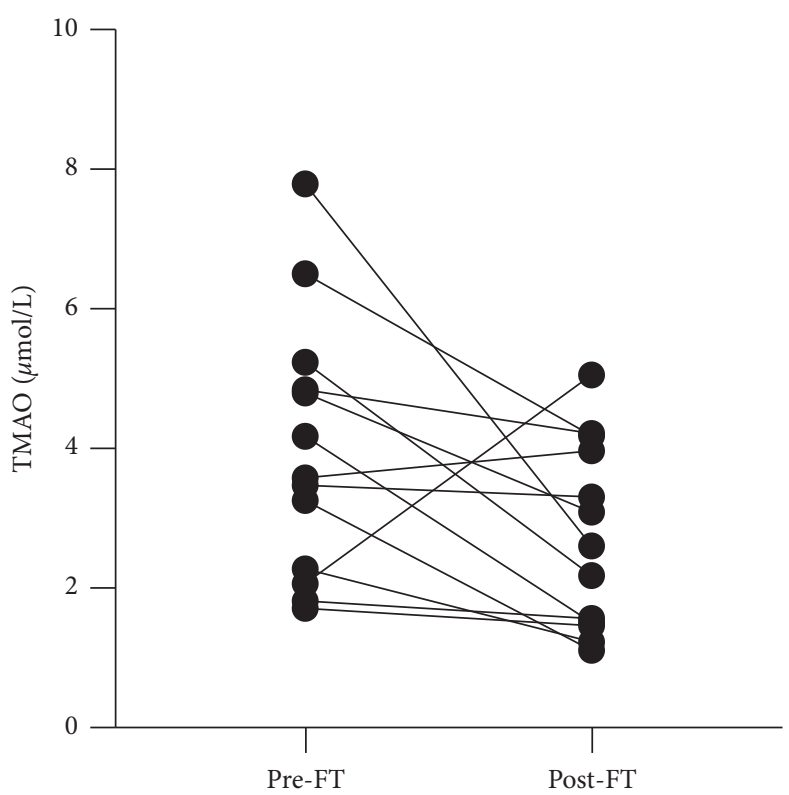

(a)

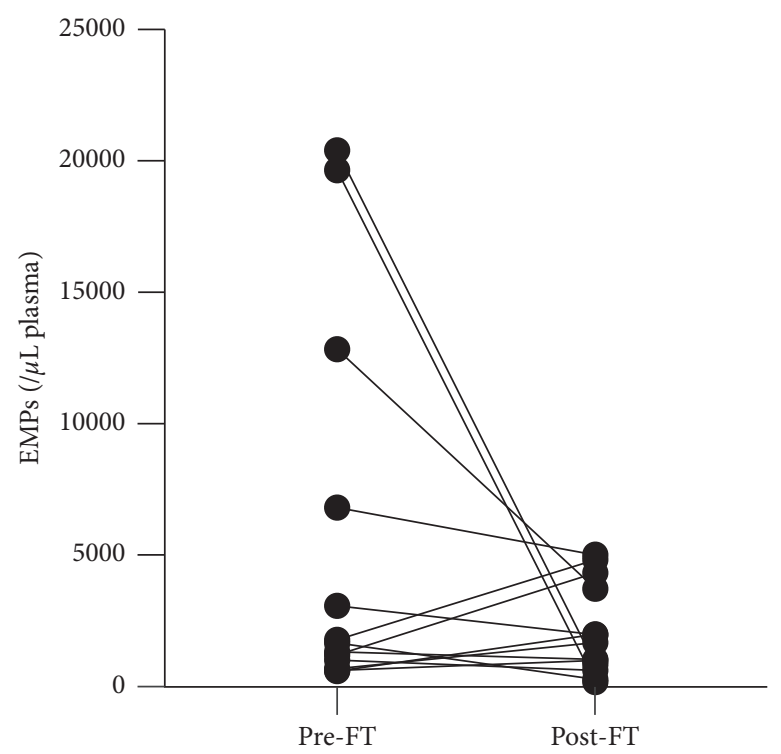

(c)

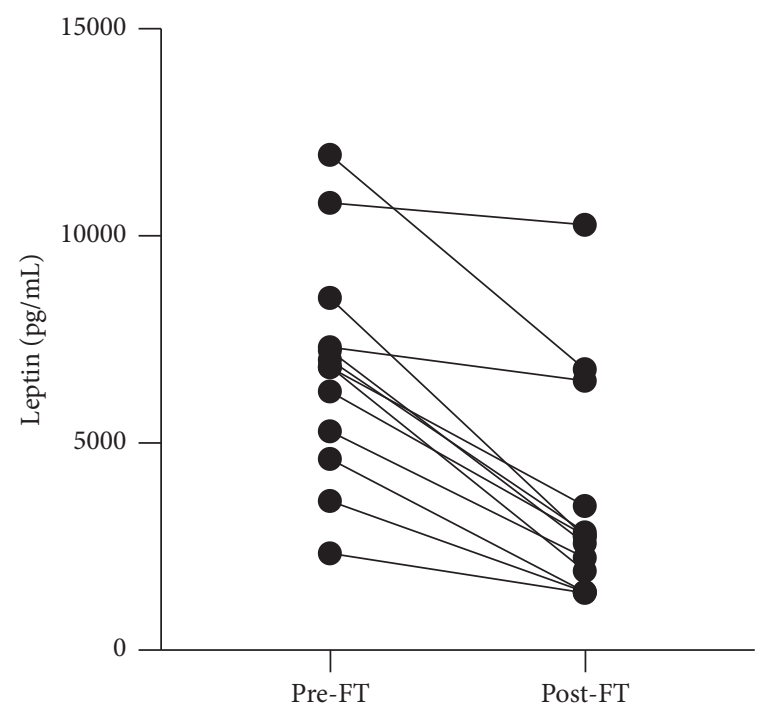

(b)

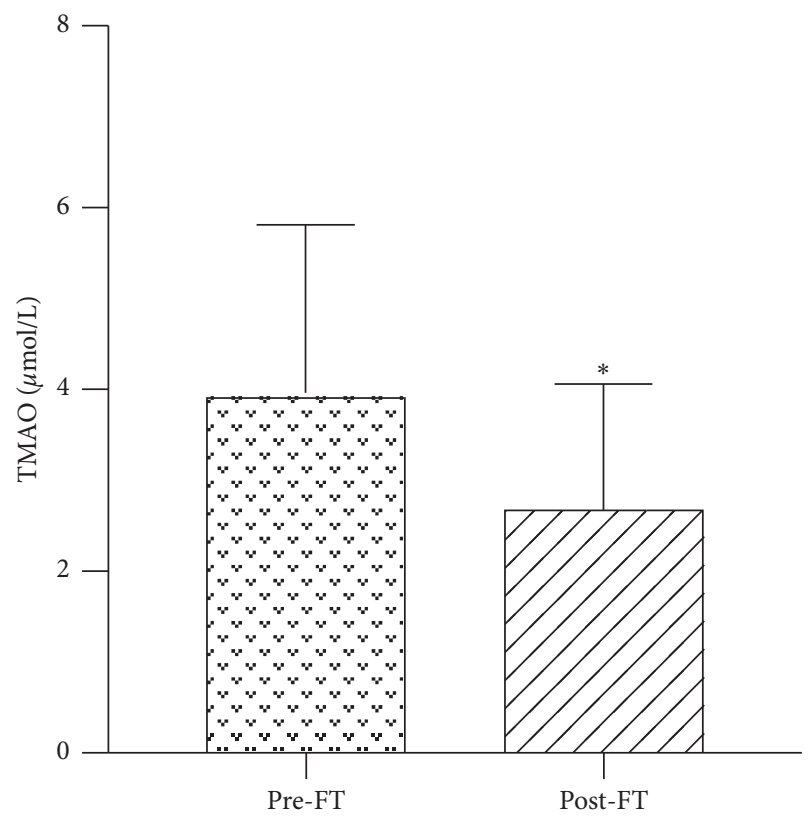

(d)

Figure 3: Continued. 


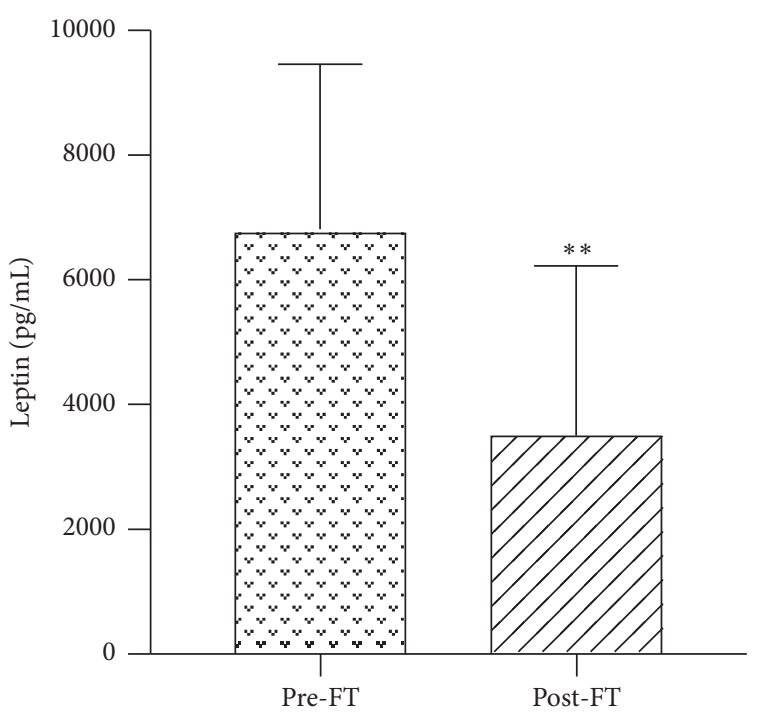

(e)

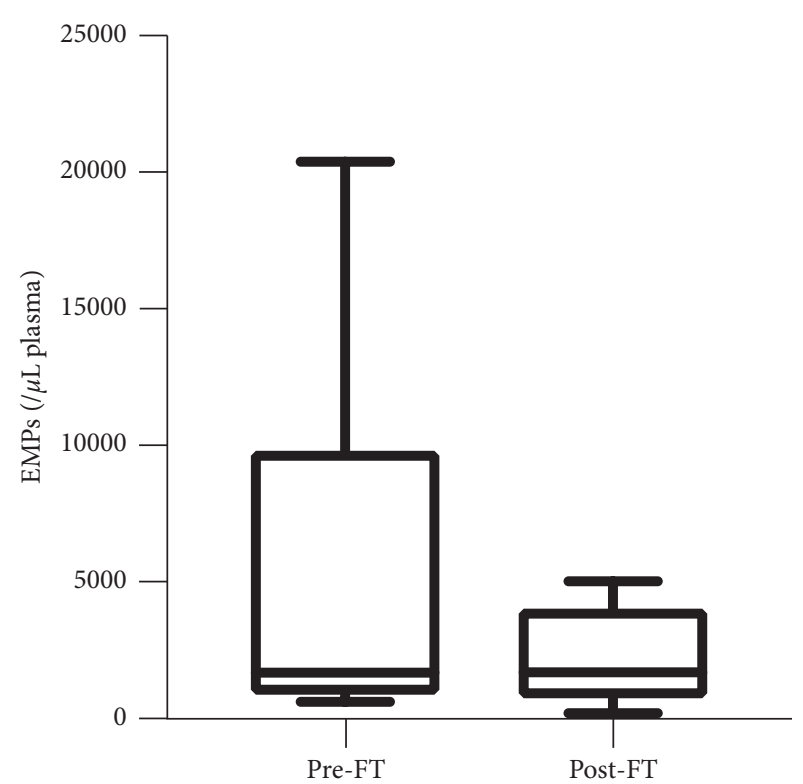

(f) Post-FT

FIgURE 3: Effects of FT on circulating TMAO, leptin, and EMPs concentrations. (a) Individual changes in TMAO. (b) Individual changes in leptin. (c) Individual changes in EMPs. (d) The mean level of TMAO. (e) The mean level of leptin. (f) Minimum to maximum EMPs (nonnormal distribution data). ${ }^{*} P<0.05,{ }^{* *} P<0.01$. TMAO: trimethylamine N-oxide; EMPs: endothelial microparticles.
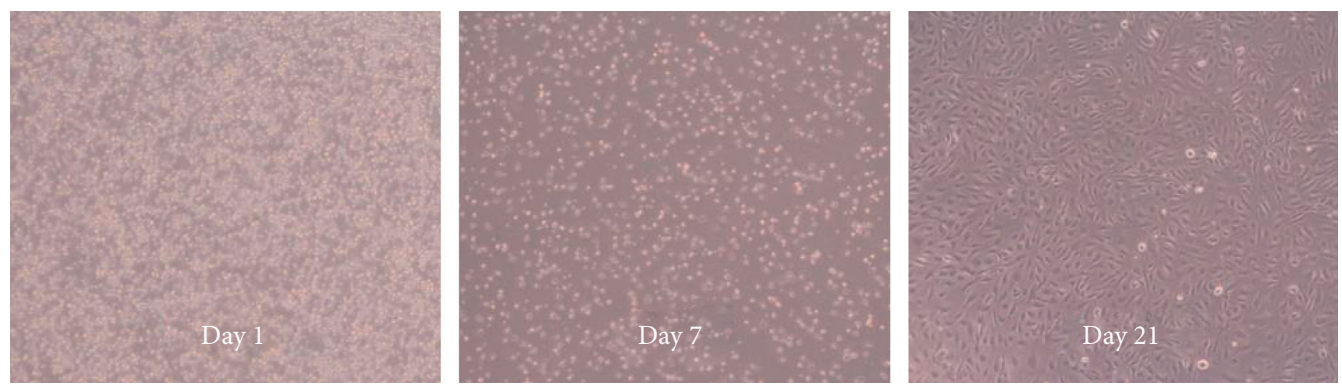

(a)
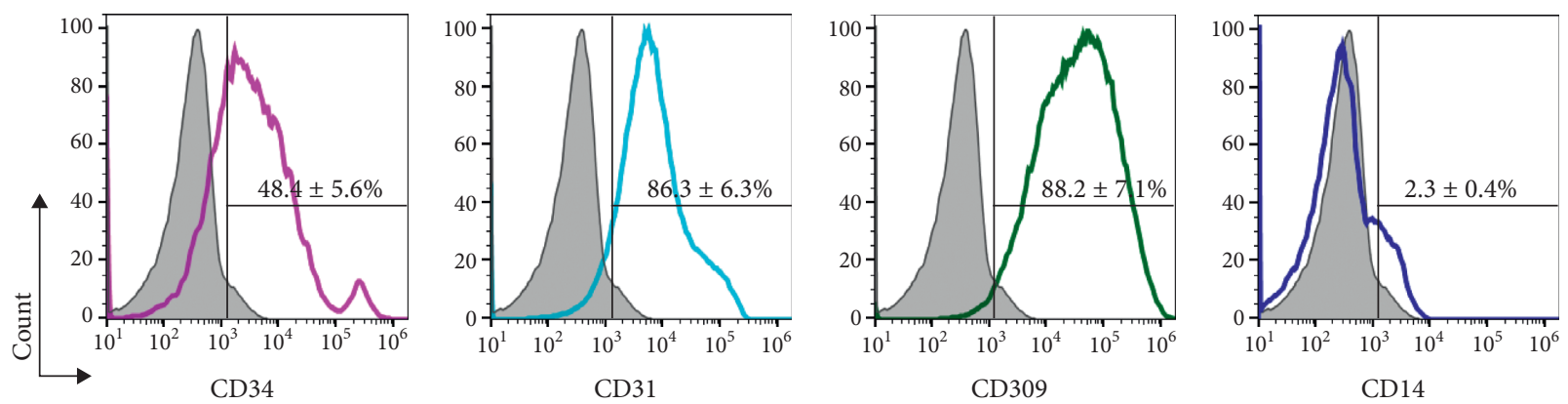

(b)

Figure 4: Identification of EPCs. (a) The morphology of late EPCs (100x magnification). (b) The late EPCs markers labeled by flow cytometry.

acids into mitochondria in order to produce energy, especially during fasting [41]. Our previous studies have proved that intravenous L-carnitine supplementation during the fasting period could ameliorate adverse reactions such as hunger and fatigue and help patients insist on the intervention $[19,20]$. In recent years, FT has been identified as a mature and effective way of very low calorie diet on weight loss and reduction of cardiovascular risk factors [19]. 

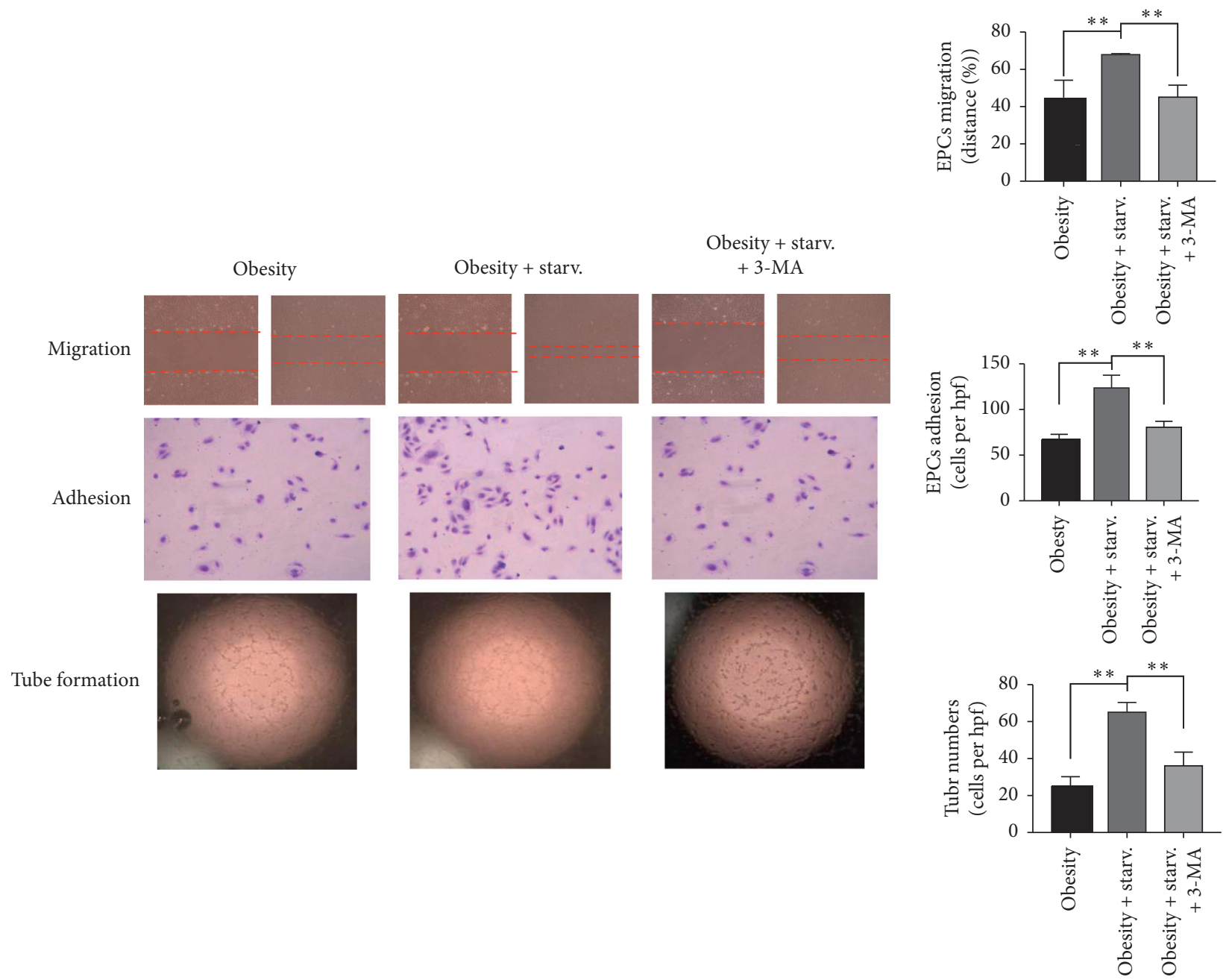

Figure 5: The function of EPCs in different conditions. EPCs with starvation showed an improvement in migration (100x magnification), adhesion (100x magnification), and tube formation (40x magnification) compared with nonstarvation group, and 3-MA blocked the beneficial effects of starvation, the effects of starvation on autophagy of EPCs in vitro.

Animal studies support the concept that calorie restriction has a beneficial impact on the vasculature. Life-long calorie restriction in mice was indicated to prevent endothelial dysfunction and arterial stiffness [42]. However, the impact of periodic fasting therapy on endothelial function in humans remains largely unknown. There were only limited data about the effect of intermittent fasting on endothelial function. An 8-week alternate day fasting study suggested a beneficial effect on insulin resistance, but not endothelial function [43]. Another 4 week 5:2 intermittent energy restriction diet strategy (two consecutive very low energy intake days) displayed no improvement on FMD [44]. The present data reported in our study showed that FMD significantly increased by about $1.26 \%$ after 7 -day fasting intervention. A notable meta-analysis shows a $13 \%$ decrease in the future risk of ASCVD for every $1 \%$ increase in FMD [45]. Hence, FT is able to significantly improve vascular endothelial function in overweight and obese individuals and might reduce the risk of ASCVD.

Although some evidence demonstrates that modest weight loss ( $8 \%$ of initial body weight) achieved with diet and lifestyle interventions seems to improve PWV [46], our study failed to see any profitable change in baPWV. As baPWV is related to many factors, especially vascular structure [47], the beneficial effect on vascular stiffness may need long-term lifestyle management.

In addition to improvements in endothelium-dependent vasodilation, FT also led to a decline in systemic levels of vascular injury-related biomarkers including TMAO and leptin. TMAO has been established as a predictor of cardiovascular events which is involved in the progress of atherogenesis, endothelial dysfunction, and metabolic disorders and significantly elevated in high BMI individuals [48]. And to our knowledge, this is the first time to find that FT significantly reduced TMAO. High level of circulating leptin enhanced the risk of ASCVD in overweight or obesity $[11,13]$ through promoting endothelial dysfunction and the expression of profibrotic markers in the heart [13]. Data in our present study also found that FT reduced serum leptin levels. Endothelial cells produce EMPs, a surrogate marker of endothelial injury, in response to cellular dysfunction and predict cardiovascular events [14, 49]. Our study showed a 


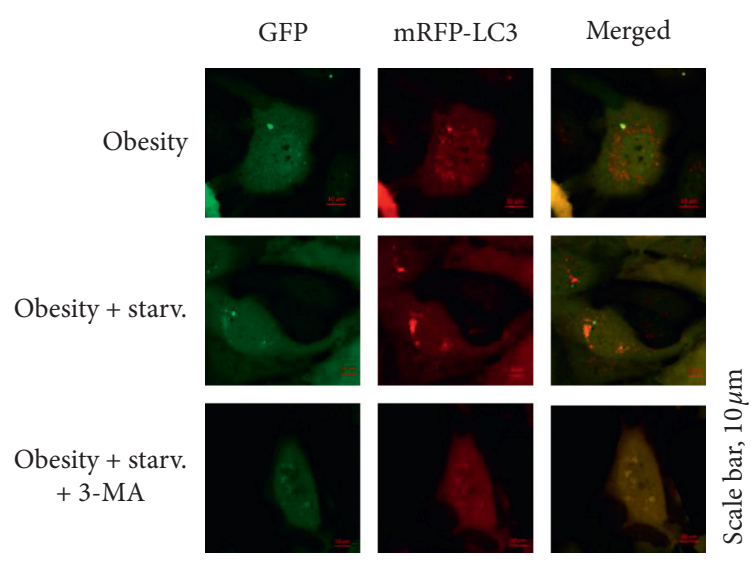

(a)

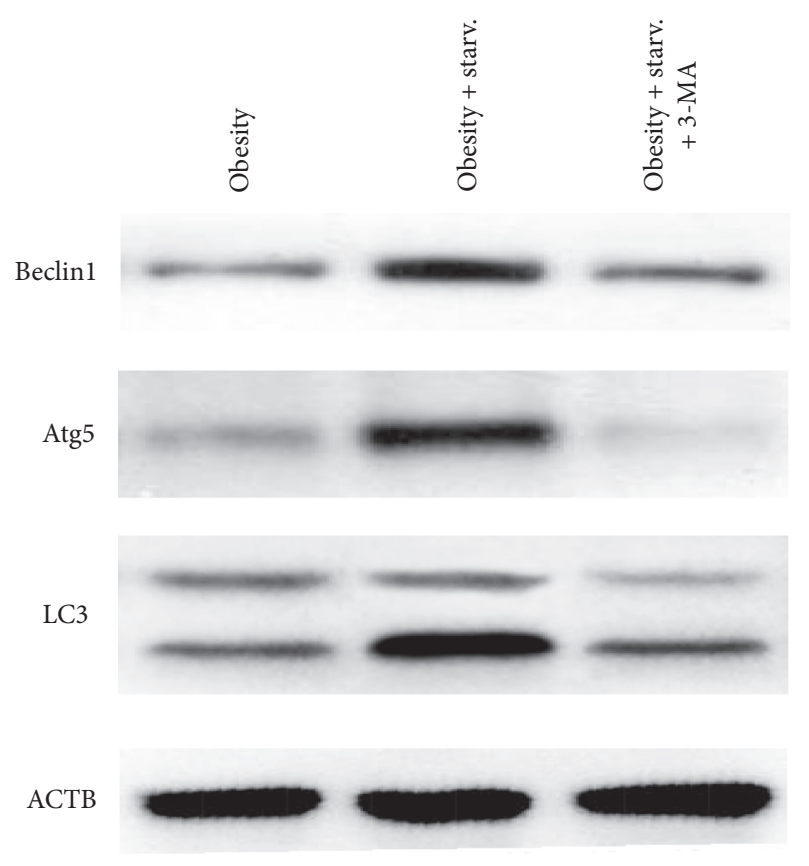

(c)

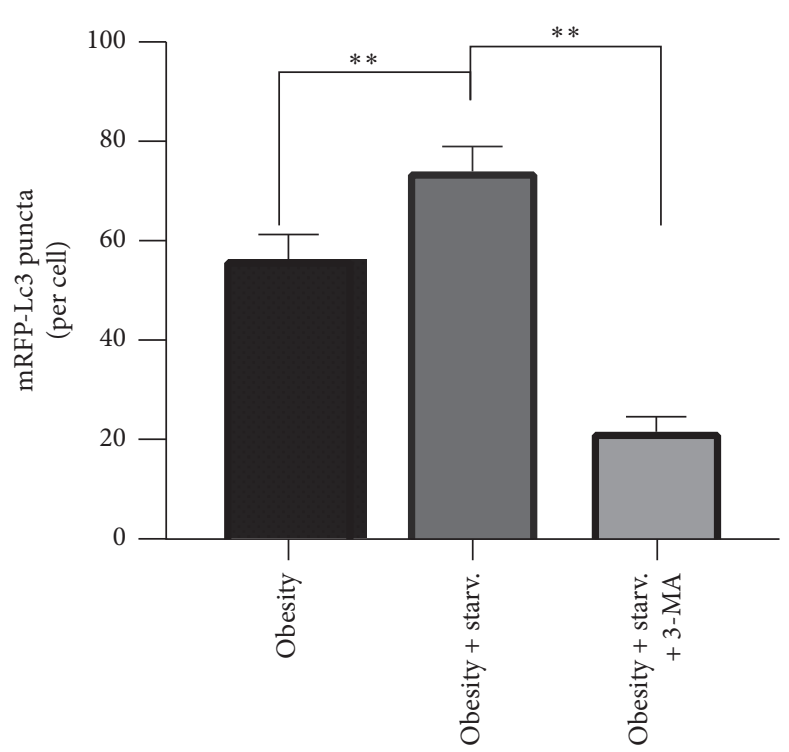

(b)

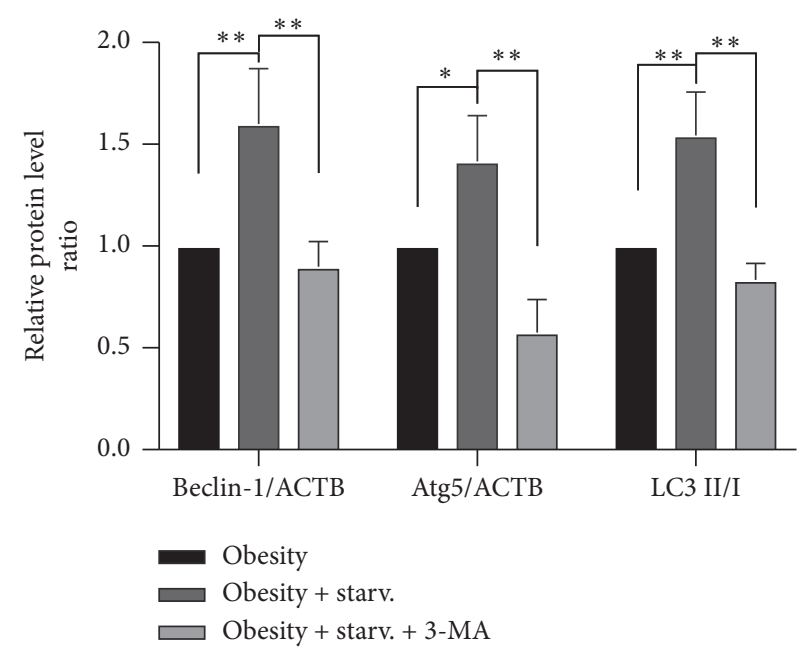

(d)

Figure 6: Autophagy level of EPCs. (a) The autophagy fluorescence intensity under a confocal microscope. (b) The mean level of fluorescence intensity. (c) The protein bands of Beclin1, Atg5, and LC3. (d) The mean level of Beclin1/ACTB, Atg5/ACTB, and LC3 II/I ratio.

descending trend on the level of EMPs whereas with no statistical significance.

Large amounts of studies demonstrate that EPCs are pivotal to the maintenance of vascular homeostasis [22] and deficiency in function and number of EPCs contribute to endothelial dysfunction in obesity $[50,51]$. There was preliminary evidence indicates that restrict calorie restriction increased endothelial function in vivo; a 2-day fasting intervention improves endothelial progenitor cell-mediated ischemic angiogenesis in mice [52]. In our study, we found starvation of obese EPCs in vitro improved the abilities of migration, adhesion, and angiogenesis.
Autophagy is a highly conserved and complex process to preserve cellular homeostasis involved in many functional proteins. Beclin-1 is a key factor in the formation of autophagy, and Atg5 and LC3 participate in the extension of the autophagic membrane to identify and wrap damaged proteins and organelles, eventually form mature autophagosomes [53]. Studies showed that autophagy defect is associated with endothelial dysfunction of patients with various metabolic disorders including obesity [26, 28]. Fasting or calorie restriction is a classical and acknowledged method to activate autophagy [29-31]. Our previous studies found that FT improved insulin resistance in obese rats by 
activating Beclin1/LC3-mediated autophagy. The present data in this study showed that starvation enhanced the fluorescence intensity of LC3 and increased the level of autophagy-related proteins such as Beclin1, Atg5, and LC3 II/I. It is worth mentioning that 3-MA, the autophagy inhibitor, blocked the beneficial effects of starvation on obese EPCs function.

The study still has several limitations. First, this work represents a small, interventional pilot study designed to determine whether FT has favorable effects on arterial function and the levels of vascular injury-related biomarkers in overweight and obese individuals. Surely, a larger, placebo-controlled randomized trial with a long time follow-up is necessary to validate our current findings. Second, whether FT would reduce the morbidity and mortality of ASCVD remains to be investigated in clinical practice. At last, the deeper mechanism of autophagy behind the favorable effects of FT on vascular homeostasis remains unclear and needs further exploration.

\section{Conclusions}

Our present work demonstrated for the first time that FT improves endothelium-dependent vasodilation and reduces vascular injury-related biomarkers in overweight and obese individuals through improving EPCs function via activating autophagy of EPCs. Overall, these findings showed that FT may be an effective therapeutic method to protect vascular health in overweight and obese individuals and retard the incidence of ASCVD in clinical practice.

\section{Data Availability}

The data used to support the findings of this study are available from the corresponding author upon request.

\section{Conflicts of Interest}

The authors declare that there are no conflicts of interest associated with the manuscript.

\section{Authors' Contributions}

Jiapan Sun and Tingying Zhang contributed equally to this work. Jiapan Sun, Tingying Zhang, and Jian Qin conceived and carried out the experiments. Li Zhang and Bin $\mathrm{Ke}$ conceived the experiments and analyzed data. Jiapan Sun and Tingying Zhang analyzed data. Each author participated in the writing part and already approved the submitted and published versions.

\section{Acknowledgments}

This work was supported by the National Nature Science Foundation of China under grant (no. 81874498); the Science and Technology Program of Guangzhou under grant (201903010044); and the Science and Shenzhen Healthcare Research Project under grant no. SZLY2018002 to Jian Qin. The authors thank the volunteers involved in this study. The authors thank professors coming from Sun Yat-sen University for their technical assistance.

\section{References}

[1] The GBD 2015 Obesity Collaborators, A. Afshin, M. H. Forouzanfar et al., "Health effects of overweight and obesity in 195 countries over 25 years," New England Journal of Medicine, vol. 377, no. 1, pp. 13-27, 2017.

[2] D. Terentes-Printzios, C. Vlachopoulos, P. Xaplanteris et al., "Cardiovascular risk factors accelerate progression of vascular aging in the general population," Hypertension, vol. 70, no. 5, pp. 1057-1064, 2017.

[3] C. Koliaki, S. Liatis, and A. Kokkinos, "Obesity and cardiovascular disease: revisiting an old relationship," Metabolism, vol. 92, pp. 98-107, 2019.

[4] F. Perticone, R. Ceravolo, M. Candigliota et al., "Obesity and body fat distribution induce endothelial dysfunction by oxidative stress: protective effect of vitamin C," Diabetes, vol. 50, no. 1, pp. 159-165, 2001.

[5] S. Masi, R. Colucci, E. Duranti et al., "Aging modulates the influence of arginase on endothelial dysfunction in obesity," Arteriosclerosis, Thrombosis, and Vascular Biology, vol. 38, no. 10, pp. 2474-2483, 2018.

[6] P. Gu and A. Xu, "Interplay between adipose tissue and blood vessels in obesity and vascular dysfunction," Reviews in Endocrine and Metabolic Disorders, vol. 14, no. 1, pp. 49-58, 2013.

[7] J. P. Zachariah, S. Hwang, N. M. Hamburg et al., "Circulating adipokines and vascular function," Hypertension, vol. 67, no. 2, pp. 294-300, 2016.

[8] Z. Wang, E. Klipfell, B. J. Bennett et al., "Gut flora metabolism of phosphatidylcholine promotes cardiovascular disease," Nature, vol. 472, no. 7341, pp. 57-63, 2011.

[9] M. M. Seldin, Y. Meng, H. Qi et al., "Trimethylamine N-oxide promotes vascular inflammation through signaling of mitogen-activated protein kinase and nuclear factor-kappaB," Journal of the American Heart Association, vol. 5, no. 2, 2016.

[10] G. Ma, B. Pan, Y. Chen et al., "Trimethylamine N-oxide in atherogenesis: impairing endothelial self-repair capacity and enhancing monocyte adhesion," Bioscience Reports, vol. 37, no. 2, 2017.

[11] S. E. Simonds, J. T. Pryor, E. Ravussin et al., "Leptin mediates the increase in blood pressure associated with obesity," Cell, vol. 159, no. 6, pp. 1404-1416, 2014.

[12] M. R. Sierra-Honigmann, A. K. Nath, C. Murakami et al., "Biological action of leptin as an angiogenic factor," Science, vol. 281, no. 5383, pp. 1683-1686, 1998.

[13] A.-C. Huby, G. Antonova, J. Groenendyk et al., "Adipocytederived hormone leptin is a direct regulator of aldosterone secretion, which promotes endothelial dysfunction and cardiac fibrosis," Circulation, vol. 132, no. 22, pp. 2134-2145, 2015.

[14] F. Dignat-George and C. M. Boulanger, "The many faces of endothelial microparticles," Arteriosclerosis, Thrombosis, and Vascular Biology, vol. 31, no. 1, pp. 27-33, 2011.

[15] F. Jansen, X. Yang, M. Hoelscher et al., "Endothelial microparticle-mediated transfer of MicroRNA-126 promotes vascular endothelial cell repair via SPRED1 and is abrogated in glucose-damaged endothelial microparticles," Circulation, vol. 128, no. 18, pp. 2026-2038, 2013.

[16] M. E. Lean, W. S. Leslie, A. C. Barnes et al., "Primary care-led weight management for remission of type 2 diabetes (DiRECT): an open-label, cluster-randomised trial," The Lancet, vol. 391, no. 10120, pp. 541-551, 2018. 
[17] S. Stekovic, S. J. Hofer, N. Tripolt et al., "Alternate day fasting improves physiological and molecular markers of aging in healthy, non-obese humans," Cell Metabolism, vol. 30, no. 3, pp. 462-476, 2019.

[18] V. D. Longo and M. P. Mattson, "Fasting: molecular mechanisms and clinical applications," Cell Metabolism, vol. 19, no. 2, pp. 181-192, 2014.

[19] J. J. Zhang, Z. B. Wu, Y. J. Cai et al., "L-carnitine ameliorated fasting-induced fatigue, hunger, and metabolic abnormalities in patients with metabolic syndrome: a randomized controlled study," Nutrition Journal, vol. 13, p. 110, 2014.

[20] T. Zhang, L. Zhang, B. Ke et al., "L-carnitine ameliorated weight loss in fasting therapy: a propensity score-matched study," Complementary Therapies in Medicine, vol. 44, pp. 162-165, 2019.

[21] T. Asahara, T. Murohara, A. Sullivan et al., "Isolation of putative progenitor endothelial cells for angiogenesis," Science, vol. 275, no. 5302, pp. 964-966, 1997.

[22] J. M. Hill, G. Zalos, J. P. J. Halcox et al., "Circulating endothelial progenitor cells, vascular function, and cardiovascular risk," New England Journal of Medicine, vol. 348, no. 7, pp. 593-600, 2003.

[23] P. R. Moreno, J. Sanz, and V. Fuster, "Promoting mechanisms of vascular health," Journal of the American College of Cardiology, vol. 53, no. 25, pp. 2315-2323, 2009.

[24] P. E. Westerweel, F. L. J. Visseren, G. R. Hajer et al., "Endothelial progenitor cell levels in obese men with the metabolic syndrome and the effect of simvastatin monotherapy vs. simvastatin/ezetimibe combination therapy," European Heart Journal, vol. 29, no. 22, pp. 2808-2817, 2008.

[25] Y. Luo, Z. Huang, J. Liao et al., "Downregulated GTCPH I/BH4 pathway and decreased function of circulating endothelial progenitor cells and their relationship with endothelial dysfunction in overweight postmenopausal women," Stem Cells International, vol. 2018, Article ID 4756263, 11 pages, 2018.

[26] A. Hammoutene, L. Biquard, J. Lasselin et al., "A defect in endothelial autophagy occurs in patients with non-alcoholic steatohepatitis and promotes inflammation and fibrosis," Journal of Hepatology, vol. 72, no. 3, pp. 528-538, 2020.

[27] M. Forte, F. Bianchi, M. Cotugno et al., "Pharmacological restoration of autophagy reduces hypertension-related stroke occurrence," Autophagy, vol. 12, pp. 1-14, 2019.

[28] S. Sciarretta, P. Zhai, D. Shao et al., "Rheb is a critical regulator of autophagy during myocardial ischemia," Circulation, vol. 125, no. 9, pp. 1134-1146, 2012.

[29] M. Bagherniya, A. E. Butler, G. E. Barreto, and A. Sahebkar, "The effect of fasting or calorie restriction on autophagy induction: a review of the literature," Ageing Research Reviews, vol. 47, pp. 183-197, 2018.

[30] X. Gao, D. Yan, Y. Zhao, H. Tao, and Y. Zhou, "Moderate calorie restriction to achieve normal weight reverses beta-cell dysfunction in diet-induced obese mice: involvement of autophagy," Nutrition \& Metabolism, vol. 12, no. 1, p. 34, 2015.

[31] C. Chang, H. Su, D. Zhang et al., "AMPK-dependent phosphorylation of GAPDH triggers Sirt1 activation and is necessary for autophagy upon glucose starvation," Molecular Cell, vol. 60, no. 6, pp. 930-940, 2015.

[32] A. E. Donald, J. P. Halcox, M. Charakida et al., "Methodological approaches to optimize reproducibility and power in clinical studies of flow-mediated dilation," Journal of the American College of Cardiology, vol. 51, no. 20, pp. 1959-1964, 2008.

[33] Z. Yang, L. Chen, C. Su et al., "Impaired endothelial progenitor cell activity is associated with reduced arterial elasticity in patients with essential hypertension," Clinical and Experimental Hypertension, vol. 32, no. 7, pp. 444-452, 2010.

[34] S. Laurent, J. Cockcroft, L. Van Bortel et al., "Expert consensus document on arterial stiffness: methodological issues and clinical applications," European Heart Journal, vol. 27, no. 21, pp. 2588-2605, 2006.

[35] M. Malik, T. M. Suboc, S. Tyagi et al., "Lactobacillus plantarum 299v supplementation improves vascular endothelial function and reduces inflammatory biomarkers in men with stable coronary artery disease," Circulation Research, vol. 123, no. 9, pp. 1091-1102, 2018.

[36] L. Bernal-Mizrachi, W. Jy, J. J. Jimenez et al., "High levels of circulating endothelial microparticles in patients with acute coronary syndromes," American Heart Journal, vol. 145, no. 6, pp. 962-970, 2003.

[37] M. A. Thomashow, D. Shimbo, M. A. Parikh et al., "Endothelial microparticles in mild chronic obstructive pulmonary disease and emphysema: the multi-ethnic study of atherosclerosis chronic obstructive pulmonary disease study," American Journal of Respiratory and Critical Care Medicine, vol. 188, no. 1, pp. 60-68, 2013.

[38] C.-H. Yoon, J. Hur, K.-W. Park et al., "Synergistic neovascularization by mixed transplantation of early endothelial progenitor cells and late outgrowth endothelial cells," Circulation, vol. 112, no. 11, pp. 1618-1627, 2005.

[39] L. Chen, M.-L. Ding, F. Wu et al., "Impaired endothelial repair capacity of early endothelial progenitor cells in hypertensive patients with primary hyperaldosteronemia," Hypertension, vol. 67, no. 2, pp. 430-439, 2016.

[40] K. M. Gadde, C. K. Martin, H.-R. Berthoud, and S. B. Heymsfield, "Obesity," Journal of the American College of Cardiology, vol. 71, no. 1, pp. 69-84, 2018.

[41] J. Pekala, B. Patkowska-Sokola, R. Bodkowski et al., "L-carnitine-metabolic functions and meaning in humans life," Current Drug Metabolism, vol. 12, no. 7, pp. 667-678, 2011.

[42] A. J. Donato, A. E. Walker, K. A. Magerko et al., "Life-long caloric restriction reduces oxidative stress and preserves nitric oxide bioavailability and function in arteries of old mice," Aging Cell, vol. 12, no. 5, pp. 772-783, 2013.

[43] K. K. Hoddy, S. Bhutani, S. A. Phillips, and K. A. Varady, "Effects of different degrees of insulin resistance on endothelial function in obese adults undergoing alternate day fasting," Nutrition and Healthy Aging, vol. 4, no. 1, pp. 63-71, 2016.

[44] M. L. Headland, P. M. Clifton, and J. B. Keogh, "Effect of intermittent energy restriction on flow mediated dilatation, a measure of endothelial function: a short report," International Journal of Environmental Research and Public Health, vol. 15, no. 6, p. 1166, 2018.

[45] Y. Inaba, J. A. Chen, and S. R. Bergmann, "Prediction of future cardiovascular outcomes by flow-mediated vasodilatation of brachial artery: a meta-analysis," The International Journal of Cardiovascular Imaging, vol. 26, no. 6, pp. 631-640, 2010.

[46] K. S. Petersen, N. Blanch, J. B. Keogh, and P. M. Clifton, "Effect of weight loss on pulse wave velocity," Arteriosclerosis, Thrombosis, and Vascular Biology, vol. 35, no. 1, pp. 243-252, 2015.

[47] C. Vlachopoulos, P. Xaplanteris, V. Aboyans et al., "The role of vascular biomarkers for primary and secondary prevention. A position paper from the European society of cardiology working group on peripheral circulation," Atherosclerosis, vol. 241, no. 2, pp. 507-532, 2015.

[48] M. L. Erickson, S. K. Malin, Z. Wang, J. M. Brown, S. L. Hazen, and J. P. Kirwan, "Effects of lifestyle intervention on plasma 
trimethylamine N-oxide in obese adults," Nutrients, vol. 11, no. 1, p. 179, 2019.

[49] H. Koga, S. Sugiyama, K. Kugiyama et al., "Elevated levels of VE-cadherin-positive endothelial microparticles in patients with type 2 diabetes mellitus and coronary artery disease," Journal of the American College of Cardiology, vol. 45, no. 10, pp. 1622-1630, 2005.

[50] C. H. Ko, S. L. Yeh, and C. L. Yeh, "Dietary supplemental glutamine enhances the percentage of circulating endothelial progenitor cells in mice with high-fat diet-induced obesity subjected to hind limb ischemia," Mediators of Inflammation, vol. 2020, Article ID 3153186, 9 pages, 2020.

[51] I. Jialal, S. Devaraj, U. Singh, and B. A. Huet, "Decreased number and impaired functionality of endothelial progenitor cells in subjects with metabolic syndrome: implications for increased cardiovascular risk," Atherosclerosis, vol. 211, no. 1, pp. 297-302, 2010.

[52] B. Xin, C. L. Liu, H. Yang et al., "Prolonged fasting improves endothelial progenitor cell-mediated ischemic angiogenesis in mice," Cellular Physiology and Biochemistry, vol. 40, no. 3-4, pp. 693-706, 2016.

[53] L. Galluzzi, E. H. Baehrecke, A. Ballabio et al., "Molecular definitions of autophagy and related processes," The EMBO Journal, vol. 36, no. 13, pp. 1811-1836, 2017. 